\title{
Audiological and otologic manifestations of glutaric aciduria type I
}

\author{
Yen-Chi Chen ${ }^{1,2 \dagger}$, Chii-Yuan Huang ${ }^{1,4}$, Yen-Ting Lee ${ }^{1}$, Chia-Hung Wu ${ }^{6,9,10}$, Sheng-Kai Chang ${ }^{5}$, Hsiu-Lien Cheng ${ }^{1,7}$, \\ Po-Hsiung Chang ${ }^{1}$, Dau-Ming Niu ${ }^{5,6^{*}}$ and Yen-Fu Cheng ${ }^{1,3,4,8^{*}}$ (D)
}

\begin{abstract}
Background: Glutaric aciduria type 1 (GA-1) is a rare disease connected with speech delay and neurological deficits. However, the audiological and otologic profiles of GA-1 have not yet been fully characterized. To our knowledge, this is the largest study of comprehensive audiological and otologic evaluation in patients with GA-1 to date.

Methods: Thirteen patients diagnosed with GA-1 between January 1994 and December 2019 with audiological, radiological and genetic manifestations were retrospectively analyzed. Hearing tests were performed in all patients. MRI was performed for radiological evaluation.

Results: Hearing loss was found in 76.9\% (10/13) of GA-1 patients, including slight hearing loss in $46.1 \%(6 / 13)$ of patients, mild hearing loss in 15.4\% (2/13) of patients, and moderate hearing loss in $7.7 \%$ (1/13) of patients. Normal hearing thresholds were seen in 23\% (3/13) of patients. Patients with intensive care unit (ICU) admission history showed significantly worse hearing than those without ( $29.17 \pm 12.47$ vs $13.56 \pm 3.93 \mathrm{~dB} \mathrm{HL}, 95 \% \mathrm{Cl} 2.92-24.70$, $p=0.0176)$. One patient had moderate sensorineural hearing loss and a past history of acute encephalopathic crisis. No usual causative gene mutations associated with hearing loss were found in these patients. MRI showed a normal vestibulocochlear apparatus and cochlear nerve. One patient with extensive injury of the basal ganglia on MRI after acute encephalopathic crisis was found to have moderate sensorineural hearing loss. Two patients with disability scores above 5 were found to have mild to moderate hearing impairment. No obvious correlation between macrocephaly and hearing loss was found.
\end{abstract}

Conclusion: A high prevalence of hearing impairment is found in GA-1 patients. Adequate audiological evaluation is essential for these patients, especially for those after encephalopathic crises or with ICU admission history.

Keywords: Glutaric aciduria type 1, Hereditary hearing loss, Syndromic hearing loss

\section{Introduction}

Glutaric aciduria type 1 (GA-1) is a rare autosomal recessive metabolic disease first described in 1975 [1]. The worldwide prevalence of GA-1 is estimated at 1:110,000,

\footnotetext{
*Correspondence: dmniu1111@yahoo.com.tw; yfcheng2@vghtpe.gov.com

${ }^{\dagger}$ Yen-Chi Chen is first author

${ }^{1}$ Department of Otolaryngology-Head and Neck Surgery, Taipei Veterans General Hospital, Taipei, Taiwan

${ }^{5}$ Department of Pediatrics, Taipei Veterans General Hospital, Taipei, Taiwan

Full list of author information is available at the end of the article
}

with approximately 500 or more cases reported to date. It is caused by mutations in the glutaryl-CoA dehydrogenase (GCDH) gene mapped to chromosome 19p13.2, which encodes a flavin adenine dinucleotide-dependent mitochondrial matrix protein that is involved in the degradation of L-lysine, L-hydroxylysine, and L-tryptophan [2]. The defect of the GCDH enzyme in the oxidative pathway leads to an accumulation of glutaric acid (GA), 3-hydroxyglutaric acid (3-OH-GA), glutaconic acid, and glutarylcarnitine (C5DC).

As GA and 3-OH-GA are putatively neurotoxic, GA-1 is characterized by neurological 
impairment, macrocephaly, dystonia and seizure [3, 4]. Without proper treatment, approximately $90 \%$ of infants will develop acute encephalopathic crisis before the age of 3 , which is during a vulnerable period of brain development [5]. According to the literature reports, patients are still susceptible to acute encephalopathic crisis even under strict treatment [6]. The diagnosis of GA-1 is made based on analyzing the C5DC concentration in dried blood spots during newborn screening and confirmed by mutations in the GCDH gene. MRI often reveals a characteristic pattern of gray and white matter abnormalities and widened cerebrospinal fluid (CSF) spaces in GA-I. A recent study described that the severity of movement is related to extensive lesions of the putamen [7]. Typically, patients present nonspecific neurologic symptoms within their infancy. Macrocephaly is a frequent (75\%) but nonspecific finding and is present at or shortly after birth. Individual speech delay and abnormal ocular findings have also been reported $[8,9]$. Neurologic sequela after crisis leads to acute bilateral striatal injury and, subsequently, a complex movement disorder. Morbidity and mortality are high in these individuals after encephalopathic crisis [5]. Treatment is analogous to that for other organic acidurias, including dietary treatment in combination with oral supplementation of L-carnitine. Mortality and morbidity are considerably reduced after proper treatment, and GA-I is therefore considered to be a treatable condition.

Most children are found to have some degree of speech difficulty (e.g., dysarthria, articulation difficulties). Psychological functions including intelligence for assessment at the general level of development, motor functions and language have been suggested. GA-1 was reported to be associated with hearing loss in isolated case reports [10]; the sparse number of reports may be due to the small patient population of GA-1 or insufficient newborn screening systems. To date, no study has explored otologic and audiological profiles in patients with GA-1. Here, we describe the audiological and otologic manifestations of 13 GA-1 patients (12 by newborn screening and one by clinical diagnosis), and all of them were retrospectively evaluated at a single tertiary referral hospital. This study is the first and largest comprehensive report to describe the otologic and auditory findings in GA-1 patients.

\section{Methods}

\section{Patients}

From January 2001 to November 2019, all patients diagnosed by newborn screening (NBS) underwent secondary confirmatory diagnosis by including quantitative analysis of GA and 3-OH-GA in urine and/or blood and $G C D H$ gene mutation analysis. Of the 16 patients who were diagnosed with GA-1 at Veterans General Hospital, 13 patients underwent comprehensive otologic evaluation and audiological tests between September 1994 and December 2019. The demographic data of the patients were retrospectively collected from their medical records, including newborn hearing screening results, age at diagnosis of GA-1, occurrence of clinical encephalopathic crisis, disability score and length of follow-up. A history of intensive care unit (ICU) admission was also recorded.

GA-1 treatment was started immediately after diagnosis. The maintenance treatment consisted of carnitine and a low lysine diet supplemented with a GA-1 special formula. Parents were given instructions regarding emergency treatment. During intercurrent illness, catabolism needs to be prevented promptly by providing a high-energy intake. Patients were seen at our clinic every 3 months. T1-weighted and T2-weighted MRI, diffusion-weighted imaging (DWI) and apparent diffusion coefficient (ADC) maps were arranged at the time of diagnosis, and new onset neurological symptoms were identified. Imaging data were not available for some patients because some parents were reluctant to subject asymptomatic children to MRI under sedation.

\section{Audiological tests}

Pure tone audiometry or conditioned behavioral audiometry (either reinforcement audiometry or conditioned play audiometry) was arranged for hearing assessment. A sound field test was conducted if the patient was not amenable to the headphone. Auditory brainstem response (ABR) was conducted for patients with poor auditory responsiveness resulting from any cognitive-related impairment. The audiological tests were conducted in a double-wall booth by an experienced audiologist. Airconduction pure tone audiometry thresholds were tested at frequencies of 250, 500, 1000, 2000, 4000, and $8000 \mathrm{~Hz}$. The pure tone average (PTA) was determined by calculating the threshold of frequencies at 500, 1000, 2000 and $4000 \mathrm{~Hz}$. Our classification of the degree of hearing loss was based on calculating the PTA. There was no hearing loss if the PTA was less than $16 \mathrm{~dB}$ HL, 16-25 dB HL was slight hearing loss, 26-40 dB HL was mild hearing loss, 41-55 dB HL was moderate hearing loss, 56-70 dB HL was moderately severe hearing loss, 71-90 dB HL was severe hearing loss, and $\geq 91 \mathrm{~dB}$ HL was profound hearing loss in accordance with the American Speech Language Hearing Association guidelines [11, 12].

Distortion product otoacoustic emissions (DPOAEs) were measured by using the Biologic Scout Sport PCbased diagnostic OAE system. The ratio of two primary tones, f2/f1, was fixed at 1.22, and moderate-level primary tones were used with $\mathrm{L} 1=65 \mathrm{~dB}$ SPL and 
$\mathrm{L} 2=55 \mathrm{~dB}$ SPL. The DPOAE measurements were over the frequency range $1-8 \mathrm{kHz}$ and interpreted as present when the signal-to-noise ratio (SNR) exceeded $6 \mathrm{~dB}$ or more at the $\mathrm{f} 2$ frequency. DPOAE was interpreted by the SNR criterion. DPOAE criteria of 6-dB DPOAE/noise ratios or better determination of the presence of DPOAE and five frequencies were required to meet the criterion classified as normal hearing.

\section{Targeted resequencing of 4 deafness-related genes (SLC26A4, GJB2, GJB3 and mtDNA 1555 and 1494) by next-generation sequencing (NGS)}

All of the patients underwent targeted sequencing of 4 common genes related to deafness in Taiwan, including SLC26A4, GJB2, GJB3 and mtDNA 1555 and 1494. First, we designed PCR primers for all candidate genes mentioned above by the online website tool Ion AmpliSeq ${ }^{\mathrm{TM}}$ Designer (Thermo Fisher). The expected sizes of all amplicons were set to $275 \mathrm{bps}$. A total amount of $10 \mathrm{ng}$ of DNA from each individual was used to construct a sample library. All designed primers were mixed according to well-optimized proportions and used for multiplex PCR. After amplification, the reactants were purified and quantified by a Qubit fluorometer (Thermo Fisher). Proper amounts of reactants were subjected to secondstage library preparation, including adapter and barcode ligation to both ends of all amplicons, and size selection (AMPure XP, Beckman Coulter). The barcoding amplicons of each individual were analyzed by a Bioanalyzer system (Agilent) to ensure the sizes and quantities of the library products. The qualified libraries were subjected to sequencing reactions by "sequencing by synthesis" (SBS) technology on a MiSeq sequencer (Illumina). The sequencing kits with different sequencing throughputs were chosen according to the amounts of library. The average depth of the target regions was more than $200 \times$. The data analysis, including quality control, reference sequence alignments, and variant annotations, was executed on the BaseSpace Sequence Hub webtool (Illumina).

\section{Disease severity}

The disability score, which is the summation of the scores for motor function, cognitive function and speech, giving a minimum of three and a maximum of nine, was developed and found to be significantly correlated with acute onset, dystonia and mortality [13]. The disability scores were simultaneously collected when the audiological test was arranged.

\section{Statistics}

The data are expressed as the mean, and descriptive statistics were also performed. Statistical analyses to compare the hearing thresholds of ICU admission, encephalopathic crisis, macroencephaly, and severe disability were performed using paired $t$ tests for normally distributed data. Statistical significance was identified when $p<0.05$. Calculations were performed using GraphPad Prism, Version 8 (GraphPad Software, CA, USA).

\section{Results \\ Patients}

The demographic data of the patients included in the study are shown in Table 1 . Thirteen patients were enrolled in this study, including 3 male and 10 female patients, ranging from 2 to 26 years old (mean: 8.0 years old). Case 13 was diagnosed clinically due to birth before the initiation of the nation-wide newborn screening for GA-1. All patients received DNA testing to confirm $G C D H$ mutations. The mean age of treatment initiation was 8.9 days from birth (excluding case 13, who started treatment at the age of 14). A total of $23.0 \%(3 / 13)$ of the patients experienced encephalopathic crisis and showed some degree of motor dysfunction.

\section{Audiological and otologic findings}

The hearing assessment was conducted with pure tone audiometry in $7(53.8 \%)$ patients and sound field tests in $5(38.5 \%)$ patients. The mean age at the first hearing test was 8.14 years old. The detailed audiometric data are shown in Table 1 . The mean AC value was $21.98 \mathrm{~dB}$ HL in all patients. Hearing thresholds were normal in $23 \%$ (3/13) of ears. Slight hearing loss was found in $46.1 \%$ $(6 / 13)$ of patients, mild hearing loss in $15.4 \%(2 / 13)$ of patients, and moderate hearing loss in $7.7 \%(1 / 13)$ of patients. All patients diagnosed with hearing loss had sensorineural hearing loss in nature. Two patients with disability scores above 5 had mild to moderate hearing impairment. In total, $46.1 \%(6 / 13)$ of patients were reported as needing ICU admission, but only half of these patients (3/6) was reported to have had an encephalopathic crisis; others were admitted due to febrile illness or seizure. None of these 6 patients received ventilation, inotropic support or hemofiltration during ICU admission. Additionally, none of these patients had received known ototoxic medication such as aminoglycoside antibiotics. The confounding of patients with ICU admission is listed in Table 2. Patients with and without ICU admission showed a significant difference in hearing loss $(29.17 \pm 12.47$ vs $13.56 \pm 3.93 \mathrm{~dB}$ HL, 95\% confidence interval (CI) 2.92-24.70, $p=0.0176$, Fig. 1), indicating worse hearing performance in patients with a history of ICU admission. A history of acute encephalopathic crisis had a negative impact on hearing performance but no statistical significance $(32.50 \pm 14.09$ vs $18.50 \pm 8.412 \mathrm{~dB}$ HL, $95 \%$ confidence interval $(\mathrm{CI})-0.04558$ to 28.05 , 


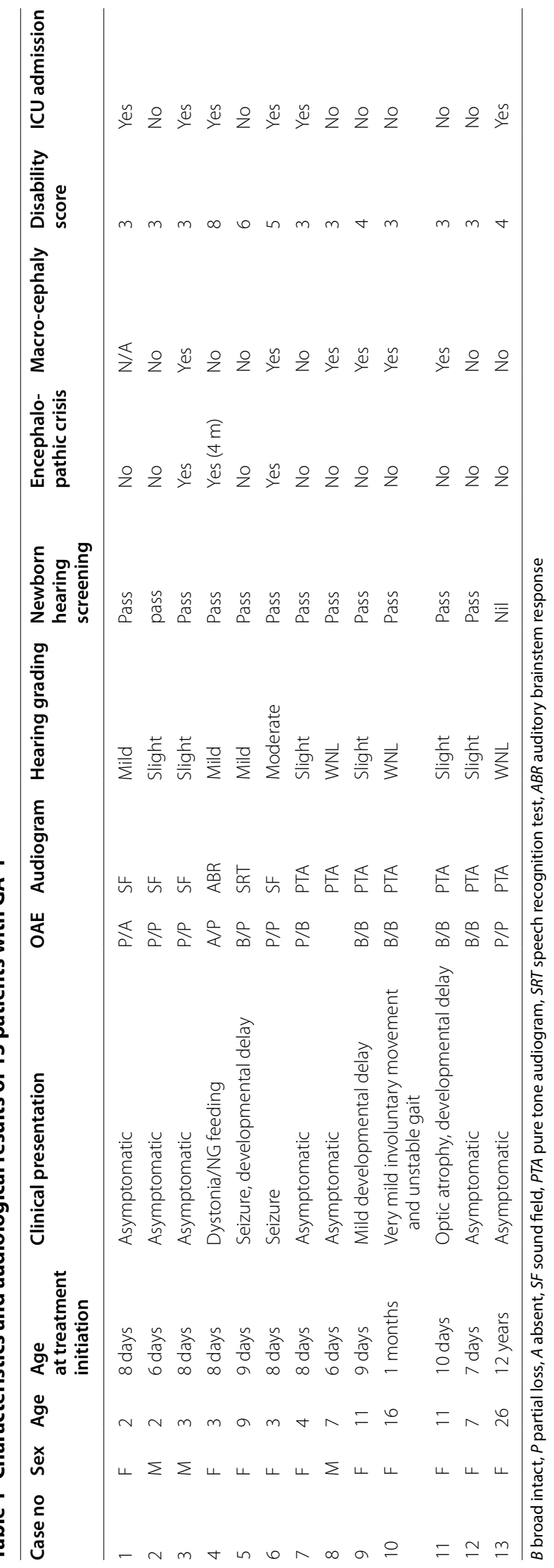


Table 2 MRI characteristics of the 13 patients

\begin{tabular}{|c|c|c|c|c|c|c|c|}
\hline Case no & $\begin{array}{l}\text { Patient's age } \\
\text { at imaging }\end{array}$ & $\begin{array}{l}\text { Widened } \\
\text { Sylvian } \\
\text { fissures }\end{array}$ & $\begin{array}{l}\text { Hyperintense T2 } \\
\text { caudate/putamen }\end{array}$ & $\begin{array}{l}\text { Presence } \\
\text { of pseudocyst }\end{array}$ & $\begin{array}{l}\text { Restricted } \\
\text { diffusion in basal } \\
\text { ganglia }\end{array}$ & $\begin{array}{l}\text { Subdural } \\
\text { hematoma } \\
\text { or hygroma }\end{array}$ & $\begin{array}{l}\text { Detectable lesion } \\
\text { along the auditory } \\
\text { pathway }\end{array}$ \\
\hline 3 & 1 year & + & + & - & + & - & - \\
\hline 5 & 12 days & + & + & - & + & - & - \\
\hline 6 & 1 year & + & + & - & + & - & - \\
\hline 7 & 18 days & + & - & - & - & - & - \\
\hline 8 & 24 days & + & + & - & - & - & - \\
\hline 9 & 10 days & + & - & - & - & - & - \\
\hline 10 & 8 years & + & - & + & + & - & - \\
\hline 11 & 4 months & + & - & - & - & - & - \\
\hline 12 & 24 days & + & - & - & + & - & - \\
\hline 13 & 1 year & + & + & - & + & - & - \\
\hline
\end{tabular}

The imaging characteristics of the 13 patients enrolled in this study are listed. In cases 1, 2 and 4, imaging data were not available in our hospital. The findings in this table were identified by MRI upon diagnosis

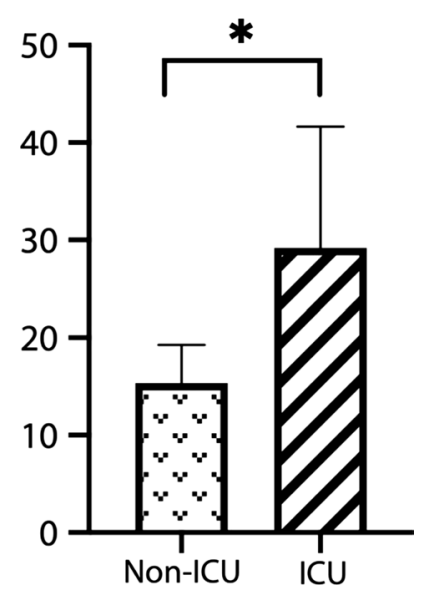

Fig. 1 Mean hearing thresholds in patients with and without an ICU admission history. Patients with a history of ICU admission showed worse hearing performance than those without (mean $\pm \mathrm{SD}=29.17 \pm 12.47$ vs $13.56 \pm 3.93 \mathrm{~dB} \mathrm{HL}, 95 \%$ confidence interval $(\mathrm{Cl})-0.04558$ to $\left.28.05, p=0.0176,{ }^{*} p<0.05\right)$

$p=0.0506)$. No obvious correlation between macrocephaly and hearing loss was found $(p=0.977)$. No otological anomalies, such as atresia of the auricle or external ear canal, otitis media with effusion or any infection or inflammatory sources, were found in these patients. DPOAEs from 12 patients showed absent or partial loss in $62.5 \%(15 / 24)$ of ears (Table 1$)$, and 11 of these patients showed slight to moderate hearing loss. The OAE results seemed highly compatible with the PTA findings.

\section{Genetic testing findings}

Genetic testing confirmed GCDH mutations in all enrolled patients. For the four deafness-related genes tested, including SLC26A4, GJB2, GJB3 and mtDNA 1555 and 1494, no pathological variants were found, indicating the origin of hearing loss from the pathological nature of GA-1.

\section{Radiological findings}

To explore the possible involvement of the auditory pathway, MRI investigations were evaluated in all thirteen patients. There was no vestibulocochlear malformation, atresia of the internal acoustic canal $(<4 \mathrm{~mm})$, or absent or hypoplastic cochlear nerve in these patients. Furthermore, no lesions were noted along the central auditory pathway, including the cochlear nucleus $(\mathrm{CN})$, superior olivary nucleus (SON), inferior colliculus (IC), medical geniculate body and auditory cortex. One of the patients (case 6) showed extensive putamen lesions on MRI (Fig. 2a-c). An oblique coronal section on T2-weighted imaging (T2WI) of patient 3 is shown as an example of comprehensive auditory pathway evaluation (Fig. 2D). The clinical presentation of the patient revealed moderate hearing loss, history of seizure attack, and impaired speech and motor functions. Table 2 shows a summary table of the MRI results and key findings for all patients.

\section{Discussion}

GA-1 is a progressive disorder that typically appears normal at birth. However, patients may experience various degrees of permanent neurological sequelae, especially after encephalopathic crisis, including motor and cognitive dysfunctions and mental retardation. Newborn screening of GA-1 started in 2001 and has been publicly offered by the National Health Insurance system in Taiwan since 2006. Our previous data revealed that the incidence of GA-1 was 1 in 106,474, which is approximately the same worldwide and in the Taiwanese population [4, 14]. To our knowledge, this is the first study to investigate 

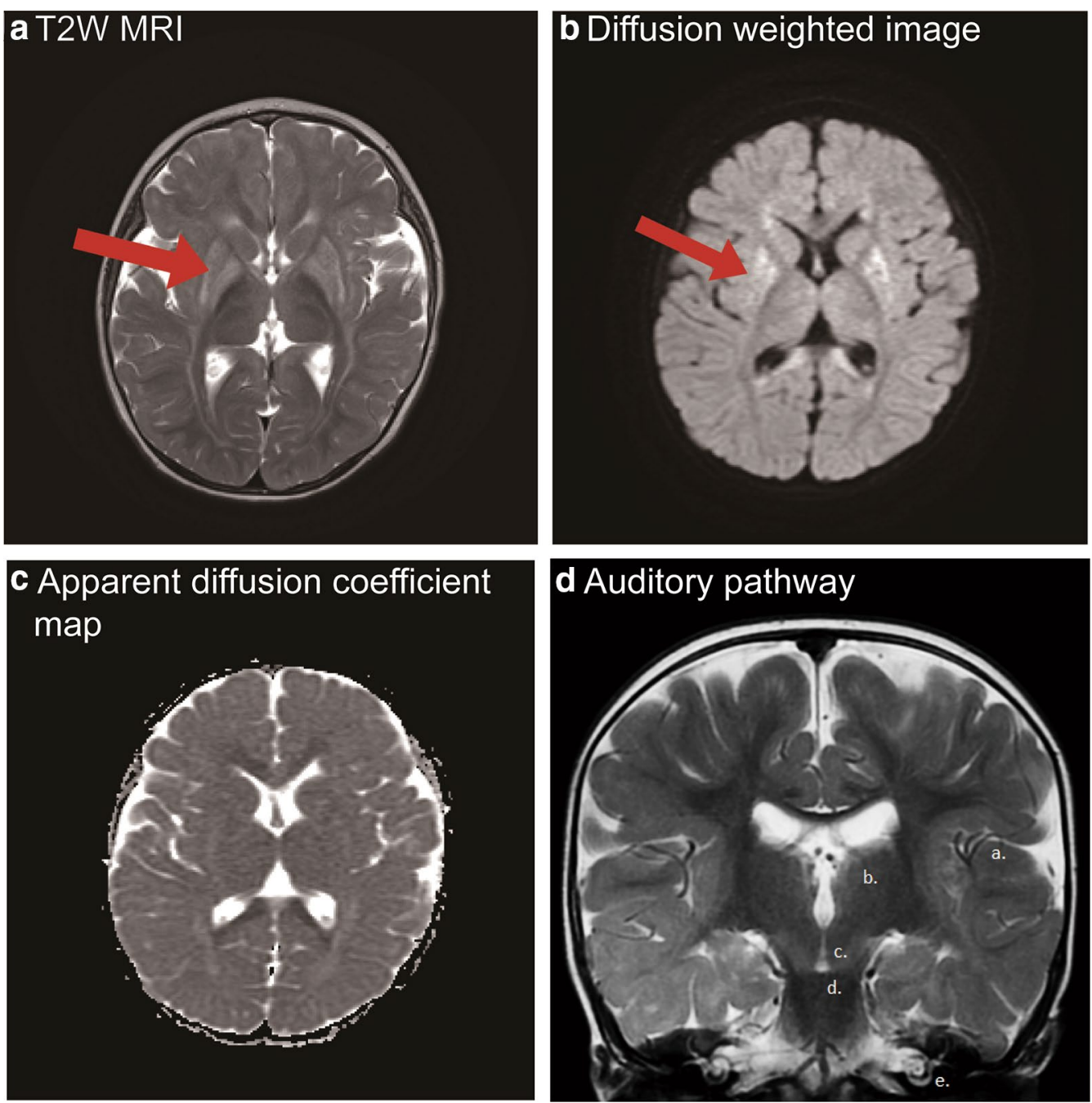

Fig. 2 MR images of patients with GA-1. A-C. The pattern of putamen injury in case 6 with a history of seizure and acute encephalopathic crisis is shown. (A) T2 weighted (T2W) MRI. The red arrow indicates extensively hyperintense striatal involvement in T2W MRI. (B) Diffusion weighted images. The red arrow reveals injury with clearly elevated diffusion of the dorsolateral putamen on diffusion weighted images. (C) Apparent diffusion coefficient map. The image shows minimal restricted diffusion affecting the bilateral putamen. (D) Illustration of imaging of the central auditory pathway. An oblique coronal section onT2WI MRI of case 3 shows the hypointense white matter tract. Letters (a) to (e) denote the apparatus of the auditory pathway. (a) Auditory cortex, (b) Thalamus (medial geniculate body), (c) Midbrain (inferior colliculus), (d) Pons (superior olivary nucleus), (e) Cochlea. Note that no space occupying lesion was noted along the tracts

the audiological and otologic profiles of GA-1. Our study may enhance awareness of early intervention in these children before dysarthria and other hearing loss-related morbidities occur (Table 3).

We found that a high percent of patients with GA-1 encountered slight to moderate sensorineural hearing loss in this study. The metabolite of GA-1 is neurotoxic, and accumulation in the central nervous system can cause severe neurological complications. The presence of 3-OH-GA in GA-1 patients may lead to increased vulnerability of endothelial structures and subsequent vascular dysfunction. The cochlea is the end organ fed solely by vessels of the labyrinthine artery, making it vulnerable to circulation causes [15]. Like other toxic metabolic diseases affecting the auditory system, the pathophysiology of hearing loss is still unclear [16]. However, it is anticipated that oxidase and neurotoxic substance accumulation in the cochlea or the rest of the auditory system causes hearing loss [17]. Other metabolic diseases such as Mitochondrial Encephalopathy, Lactic Acidosis, and Stroke-like episodes (MELAS), homocystinuria, mucopolysaccharidosis and congenital hyperlactic acidemia were also reported directly or indirectly related to hearing loss [18-23]. Several lysosomal storage disorders 


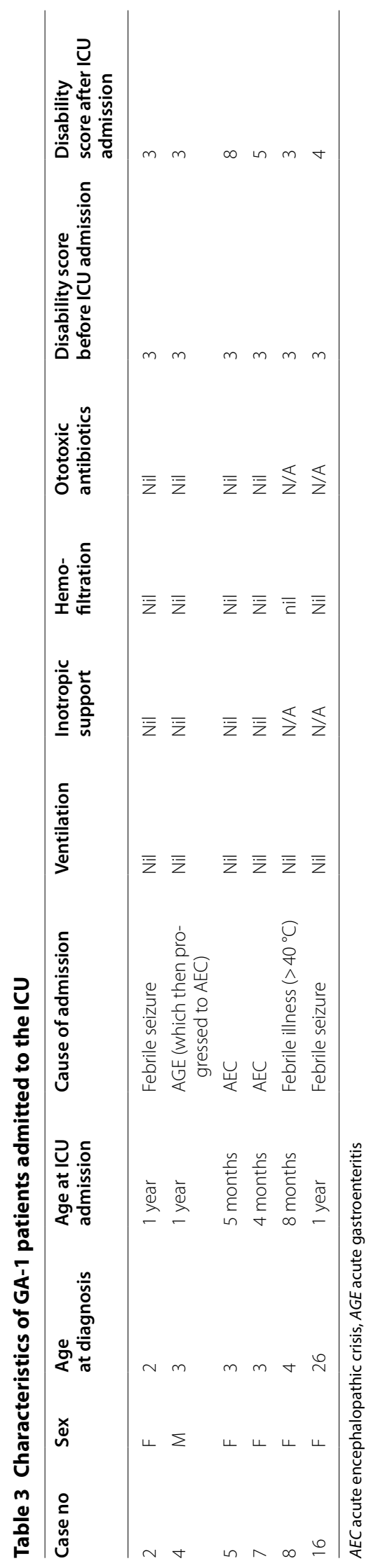


have been reported to affect hearing functions in the pediatric population. For example, in classic Fabry disease patients, it was postulated that globotriaosylsphingosine (lyso-Gb3), a neurotoxic substance predominantly deposited in these patients, causes stria vascularis injury and spiral ligament atrophy [24]. In the animal model of Pompe disease, glycogen accumulation was found in the cochlea, affecting inner and outer cells and finally leading to hearing loss [25]. An animal model of GA-1 (Gcdh ${ }^{-1-}$ mice), demonstrated typical motor deficits and diffuse spongiform myelinopathy, but did not seem to show any hearing phenotypes [26]. While hearing loss is suspected to be caused by the accumulation of toxic metabolites in the auditory pathway, including the cochlea, auditory nerve, and brainstem, further studies are needed to elucidate the causes.

We found that $75 \%$ of GA-1 patients had varying degrees of hearing loss in our study. The high prevalence of hearing loss was not reported previously. OAEs showed partial missing signals, indicating partial loss of function of the outer hair cells in the cochlea. It has been shown that over $50 \%$ of prelingual hearing loss has attributable genetic factors [27]. To preclude other possible genetic causes of hearing loss, the four most common deafness-associated mutations in the Taiwanese population, GJB2, SLC26A4, GJB3, and mitochondrial $m .1555 A>G$, were included in our NGS panel. In terms of the allele frequency in the hearing-impaired population, these 4 mutations account for over $80 \%$ of the known deafness-associated mutations in Taiwan [28]. We did not find any pathologic variants of the four genes identified in our study. The inclusion of the 4 common deafness-associated genes in the genetic tests offers strong evidence to preclude hearing loss caused by the popular deafness-related gene variants in the Taiwanese population.

MRI offers the advantage of detecting causes of sensorineural hearing loss, including large vestibular aqueduct, inner ear anomaly, cochlear nerve deficiency and postinfection changes [29]. In our study, all MRI series showed negative findings of the peripheral auditory apparatus, including the vestibulocochlear apparatus, cochlear nerve and endolymphatic duct, or the central auditory pathway. As such, the hearing impairment of children with GA-1 was not structural or caused by infections related to the peripheral or central auditory organs. In addition, the DWI and ADC maps have value for differentiating cytotoxic brain injury from vasogenic brain edema [30]. We found that most of the brain edema in our patients was cytotoxic edema. It was postulated that $76 \%$ of patients with GA- 1 and $88 \%$ after encephalopathic crisis have injury over the putamen on MRI [31]. We found that one patient with acute encephalopathic crisis, who had moderate sensorineural hearing loss, had extensive injury of the basal ganglia on MRI. The relationship between hearing loss and cytotoxic brain injury requires further clarification in the future.

The evolution of diffusion tensor imaging in MRI has led to the finding of an association between sensorineural hearing loss and white matter tract microstructure [32]. Some extensive functional MR sequences, including MR spectroscopy, have been proposed to examine patients with GA-1 [33]. Fractional anisotropy (FA) is widely used as an important parameter to change the functional integrity of white matter. The central auditory pathway, including the auditory cortex and IC, was found to have significantly lower FA values than normal hearing subjects, which is widely used as an important parameter to determine the directionality of diffusion [34]. However, most parents are reluctant to allow the patients receive these functional sequences because the prolonged general anesthetic duration in pediatric patients may increase the risk of neurodevelopmental effects [35]. It has also been reported that the microstructures of the brain have clinical value in the determination of speech and language therapy [36]. It is of interest to further determine the structural or functional changes within the auditory pathway among GA-1 patients with hearing impairment in the future.

In our study, hearing loss was found in $69.2 \%$ of patients, which is much higher than the global prevalence of pediatric hearing impairment $(7.6-16.4 \%$ worldwide [37-39]). However, all of our patients passed the newborn hearing screening, implying that onset hearing loss may not occur at birth but instead during later childhood. It is well-recognized that hearing is critical for speech development in the early years of a child's life, and even mild sensorineural hearing loss may also cause a negative impact on the academic performance or language skills of children [40-43]. GA-1 was reported to affect fine motor skills and speech, and early intervention and rehabilitation were suggested to optimize the educational environment for patients with GA-1 [6, 44, 45]. As there is no report regarding the audiological and otologic manifestation of GA-1, a routine hearing test is not included in the consensus guidelines [6]. Based on this study, we suggest routine audiological and speech screening after birth for GA-1 patients.

Some risk factors have been reported to affect hearing performance, such as ICU admission, ototoxic drug use, genetic syndromes and recurrent middle ear infection $[38,46]$. In our study, hearing loss was found in 5 of 6 patients with a history of ICU admission. In addition, a significantly higher average hearing threshold was also found in patients with a history of ICU admission. Previous studies showed that the prevalence of hearing loss in 
the ICU population was $3.2 \%$, which is significantly lower than our result $[47,48]$. While several factors have been reported to be associated with poor hearing in patients with ICU admittance, such as sepsis, meningitis, cerebral bleeding, antibiotic use and noise exposure [48, 49], further studies are needed to explore the underlying reasons for poor hearing performance in those with a history of ICU admission.

Although the results of the present study showed a high prevalence of hearing impairment among these children, there were several limitations, such as the nature of retrospective and cross-sectional observational studies, the small number of patients, and the use of a single medical facility. Therefore, data on other predisposing factors leading to hearing loss could not be fully collected, such as the use of ototoxic medications, noise exposure, infections during pregnancy and trauma. Furthermore, the origins of sensorineural hearing loss in patients with GA-1 also require further neuropathological and/ or radiological studies. Nevertheless, this study provides the first evidence that children with GA-1 have a high prevalence of abnormal hearing performance, and further studies are needed to address rehabilitative and educational issues.

\section{Conclusion}

Hearing loss is a common issue in patients with GA-1. Clinicians should consider the high prevalence of hearing loss in these patients, especially in patients with encephalopathic crisis or ICU admittance. Further studies are needed to investigate the nature of hearing impairment in GA-1.

\section{Abbreviations}

GA-1: Glutaric aciduria type 1; PTA: Pure tone audiometry; DPOAE: Distortion product otoacoustic emissions; ABR: Auditory brainstem response; NBS: Newborn screening.

\section{Acknowledgements}

The authors acknowledge Shang-Liang Wu, Ph.D., for the statistical analyses.

\section{Authors' contributions}

YCC, YFC and CYH participated in writing the final draft of the manuscript. YCC and YTL designed the study, interpreted the results, and wrote a draft of the manuscript. DMN and YFC conceptualized and designed the study, interpreted the data, and critically revised the manuscript. SKC and HLC reviewed the raw data and revised the descriptions. CHW helped review all images in our study.

\section{Availability of data and materials \\ Not applicable.}

\section{Ethics approval and consent to participate}

The trial was approved by the Institutional Review Board of Taipei Veterans General Hospital.

\section{Consent for publication}

Not applicable.

\section{Competing interests}

The authors declare that they have no competing interests.

\section{Author details}

${ }^{1}$ Department of Otolaryngology-Head and Neck Surgery, Taipei Veterans General Hospital, Taipei, Taiwan. ${ }^{2}$ Department of Otolaryngology-Head and Neck Surgery, Kaoshiung Municipal Gangshan Hospital (Outsourceded by Show-Chwan Memorial Hospital), Kaoshiung, Taiwan. ${ }^{3}$ Department of Medical Research, Taipei Veterans General Hospital, Taipei, Taiwan. ${ }^{4}$ Department of Otolaryngology-Head and Neck Surgery, School of Medicine, National Yang-Ming University, Taipei, Taiwan. ${ }^{5}$ Department of Pediatrics, Taipei Veterans General Hospital, Taipei, Taiwan. ${ }^{6}$ Institute of Clinical Medicine, National Yang-Ming University, Taipei, Taiwan. ${ }^{7}$ Department of Biomedical Engineering, National Yang-Ming University, Taipei, Taiwan. ${ }^{8}$ Institute of Brain Science, National Yang-Ming University, Taipei, Taiwan. ${ }^{9}$ Department of Radiology, Taipei Veterans General Hospital, Taipei, Taiwan. ${ }^{10}$ School of Medicine, National Yang-Ming University, Taipei, Taiwan.

Received: 9 May 2020 Accepted: 5 October 2020

Published online: 01 December 2020

\section{References}

1. Goodman SI, Markey SP, Moe PG, Miles BS, Teng CC. Glutaric aciduria; a"new" disorder of amino acid metabolism. Biochem Med. 1975;12(1):12-21.

2. Fu Z, Wang M, Paschke R, Rao KS, Frerman FE, Kim J-JP. Crystal structures of human glutaryl-CoA dehydrogenase with and without an alternate substrate: structural bases of dehydrogenation and decarboxylation reactions. Biochemistry. 2004;43(30):9674-84.

3. Kölker S, Sauer S, Surtees R, Leonard J. The aetiology of neurological complications of organic acidaemias - a role for the blood-brain barrier. J Inherit Metab Dis. 2006;29(6):701-4.

4. Kölker S, Garbade SF, Boy N, et al. Decline of acute encephalopathic crises in children with glutaryl-CoA dehydrogenase deficiency identified by newborn screening in Germany. Pediatr Res. 2007;62(3):357.

5. Kölker S, Garbade SF, Greenberg CR, et al. Natural history, outcome, and treatment efficacy in children and adults with glutaryl-CoA dehydrogenase deficiency. Pediatr Res. 2006;59(6):840.

6. Boy N, Mühlhausen C, Maier EM, et al. Proposed recommendations for diagnosing and managing individuals with glutaric aciduria type l: second revision. J Inherit Metab Dis. 2017;40(1):75-101.

7. Boy N, Garbade SF, Heringer J, Seitz A, Kölker S, Harting I. Patterns, evolution, and severity of striatal injury in insidious-vs acute-onset glutaric aciduria type 1.J Jnherit Metab Dis. 2019;42(1):117-27.

8. Sattiavany V, Bhargavi P, John S. Language characteristics in child with glutaric acidemia Type 1. Indian J Otol. 2016;22(4):288.

9. Gago LC, Wegner RK, Capone A Jr, Williams GA. Intraretinal hemorrhages and chronic subdural effusions: glutaric aciduria type 1 can be mistaken for shaken baby syndrome. Retina. 2003;23(5):724-6.

10. Park JD, Lim B, Kim KJ, et al. Glutaric aciduria type 1 in Korea: report of two novel mutations. J Korean Med Sci. 2010;25(6):957-60.

11. Holmes AE, Niskar AS, Kieszak SM, Rubin C, Brody DJ. Mean and median hearing thresholds among children 6 to 19 years of age: The Third National Health and Nutrition Examination Survey, 1988 to 1994, United States. Ear Hear. 2004:25(4):397-402.

12. Lieu JEC. Speech-language and educational consequences of unilateral hearing loss in children. Arch Otolaryngol Head Neck Surg. 2004;130(5):524-30

13. Kyllerman M, Skjeldal O, Christensen E, et al. Long-term follow-up, neurological outcome and survival rate in 28 Nordic patients with glutaric aciduria type 1. Eur J Paediatr Neurol. 2004;8(3):121-9.

14. Tsai $\mathrm{F}-\mathrm{C}$, Lee $\mathrm{H}-\mathrm{J}$, Wang A-G, et al. Experiences during newborn screening for glutaric aciduria type 1: diagnosis, treatment, genotype, phenotype, and outcomes. J Chin Med Assoc. 2017:80(4):253-61.

15. Mohammed SH, Shab-Bidar S, Abuzerr S, Habtewold TD, Alizadeh S, Djafarian K. Association of anemia with sensorineural hearing loss: a systematic review and meta-analysis. BMC Res Notes. 2019;12(1):283. 
16. Kamogashira T, Fujimoto C, Yamasoba T. Reactive oxygen species, apoptosis, and mitochondrial dysfunction in hearing loss. BioMed Res Int. 2015;2015:617207. https://doi.org/10.1155/2015/617207.

17. Pudrith C, Dudley WN. Sensorineural hearing loss and volatile organic compound metabolites in urine. Am J Otolaryngol. 2019;40(3):409-12

18. Di Stadio A, Pegoraro V, Giaretta L, Dipietro L, Marozzo R, Angelini C. Hearing impairment in MELAS: new prospective in clinical use of microRNA, a systematic review. Orphanet J Rare Dis. 2018;13(1):35.

19. Tinelli C, Di Pino A, Ficulle E, Marcelli S, Feligioni M. Hyperhomocysteinemia as a risk factor and potential nutraceutical target for certain pathologies. Front Nutr. 2019;6:49.

20. Gökdoğan Ç, Altinyay S, Gökdoğan O, et al. Audiologic evaluations of children with mucopolysaccharidosis. Braz J Otorhinolaryngol. 2016;82(3):281-4.

21. Kon K, Inagaki M, Kaga M, Sasaki M, Hanaoka S. Otoacoustic emission in patients with neurological disorders who have auditory brainstem response abnormality. Brain Dev. 2000;22(5):327-35.

22. Alonso-Varela M, Gil-Peña H, Coto E, et al. Distal renal tubular acidosis, clinical manifestations in patients with different underlying gene mutations. Pediatr Nephrol. 2018;33(9):1523-9.

23. Partearroyo T, Vallecillo N, Pajares MA, Varela-Moreiras G, Varela-Nieto I. Cochlear homocysteine metabolism at the crossroad of nutrition and sensorineural hearing loss. Front Mol Neurosci. 2017;10:107.

24. Suntjens EB, Smid BE, Biegstraaten M, Dreschler WA, Hollak CE, Linthorst GE. Hearing loss in adult patients with Fabry disease treated with enzyme replacement therapy. J Inherit Metab Dis. 2015;38(2):351-8.

25. Kamphoven $\mathrm{JH}$, de Ruiter MM, Winkel LP, et al. Hearing loss in infantile Pompe's disease and determination of underlying pathology in the knockout mouse. Neurobiol Dis. 2004;16(1):14-20.

26. Koeller DM, Woontner M, Crnic LS, et al. Biochemical, pathologic and behavioral analysis of a mouse model of glutaric acidemia type I. Hum Mol Genet. 2002;11(4):347-57.

27. Schrijver I. Hereditary non-syndromic sensorineural hearing loss: transforming silence to sound. J Mol Diagn. 2004;6(4):275-84.

28. Wu C-C, Chen P-J, Chiu Y-H, Lu Y-C, Wu M-C, Hsu C-J. Prospective mutation screening of three common deafness genes in a large Taiwanese Cohort with idiopathic bilateral sensorineural hearing impairment reveals a difference in the results between families from hospitals and those from rehabilitation facilities. Audiol Neurotol. 2008;13(3):172-81.

29. Prosser JD, Cohen AP, Greinwald JH. Diagnostic evaluation of children with sensorineural hearing loss. Otolaryngol Clin North Am. 2015:48(6):975-82.

30. Poretti A, Blaser $\mathrm{SI}$, Lequin $\mathrm{MH}$, et al. Neonatal neuroimaging findings in inborn errors of metabolism. J Magn Reson Imaging. 2013;37(2):294-312.

31. Mohammad SA, Abdelkhalek HS, Ahmed KA, Zaki OK. Glutaric aciduria type 1: neuroimaging features with clinical correlation. Pediatr Radiol. 2015;45(11):1696-705.

32. Chang Y, Lee S-H, Lee Y-J, et al. Auditory neural pathway evaluation on sensorineural hearing loss using diffusion tensor imaging. NeuroReport. 2004;15(11):1699-703.

33. Pérez-Dueñas B, De La Osa A, Capdevila A, et al. Brain injury in glutaric aciduria type l: the value of functional techniques in magnetic resonance imaging. Eur J Paediatr Neurol. 2009;13(6):534-40.
34. Tarabichi O, Kozin ED, Kanumuri W, et al. Diffusion tensor imaging of central auditory pathways in patients with sensorineural hearing loss: a systematic review. Otolaryngol Head Neck Surg. 2018;158(3):432-42.

35. Bartels DD, McCann ME, Davidson AJ, Polaner DM, Whitlock EL, Bateman BT. Estimating pediatric general anesthesia exposure: quantifying duration and risk. Pediatr Anesth. 2018;28(6):520-7.

36. Rachakonda T, Shimony JS, Coalson RS, Lieu JE. Diffusion tensor imaging in children with unilateral hearing loss: a pilot study. Front Syst Neurosci. 2014;8:87.

37. Niskar AS, Kieszak SM, Holmes A, Esteban E, Rubin C, Brody DJ. Prevalence of hearing loss among children 6 to 19 years of age: the Third National Health and Nutrition Examination Survey. JAMA. 1998;279(14):1071-5.

38. le Clercq CM, Van Ingen $G$, Ruytjens $L$, et al. Prevalence of hearing loss among children 9 to 11 years old: the generation R study. JAMA Otolaryngol Head Neck Surg. 2017;143(9):928-34.

39. Lieu JE. Variations in the prevalence of hearing loss in children: truth or artifact? JAMA Otolaryngol Head Neck Surg. 2017;143(9):935-6.

40. Carney AE, Moeller MP. Treatment efficacy: hearing loss in children. J Speech Lang Hear Res. 1998;41(1):S61-84.

41. Hicks CB, Tharpe AM. Listening effort and fatigue in school-age children with and without hearing loss. J Speech Lang Hear Res. 2002:45(3):573-84.

42. Davis JM, Elfenbein J, Schum R, Bentler RA. Effects of mild and moderate hearing impairments on language, educational, and psychosocial behavior of children. J Speech Hear Disord. 1986;51(1):53-62.

43. Halliday LF, Tuomainen O, Rosen S. Language development and impairment in children with mild to moderate sensorineural hearing loss. J Speech Lang Hear Res. 2017;60(6):1551-67.

44. Brown A, Crowe L, Beauchamp MH, Anderson V, Boneh A. Neurodevelopmental profiles of children with glutaric aciduria type I diagnosed by newborn screening: a follow-up case series. In: JIMD reports, vol. 18. Berlin: Springer; 2014. p. 125-34.

45. McKay S, Gravel JS, Tharpe AM. Amplification considerations for children with minimal or mild bilateral hearing loss and unilateral hearing loss. Trends Amplif. 2008;12(1):43-54.

46. Bielecki I, Horbulewicz A, Wolan T. Risk factors associated with hearing loss in infants: an analysis of 5282 referred neonates. Int J Pediatr Otorhinolaryngol. 2011;75(7):925-30.

47. Hille ET, Van Straaten H, Verkerk PH. Prevalence and independent risk factors for hearing loss in NICU infants. Acta Paediatr. 2007:96(8):1155-8.

48. Coenraad S, Goedegebure A, Van Goudoever J, Hoeve L. Risk factors for sensorineural hearing loss in NICU infants compared to normal hearing NICU controls. Int J Pediatr Otorhinolaryngol. 2010;74(9):999-1002.

49. Carvalho WB, Pedreira ML, de Aguiar MAL. Noise level in a pediatric intensive care unit. J Pediatr (Rio J). 2005;81 (6):495-8.

\section{Publisher's Note}

Springer Nature remains neutral with regard to jurisdictional claims in published maps and institutional affiliations.

Ready to submit your research? Choose BMC and benefit from

- fast, convenient online submission

- thorough peer review by experienced researchers in your field

- rapid publication on acceptance

- support for research data, including large and complex data types

- gold Open Access which fosters wider collaboration and increased citations

- maximum visibility for your research: over $100 \mathrm{M}$ website views per year

At BMC, research is always in progress.

Learn more biomedcentral.com/submissions 